\title{
Psychosocial dimensions of Paediatric Cardiology - more than statistics and testing?
}

\author{
Henri A. Verhaaren \\ Department of Paediatric Cardiology, University Hospital, Ghent, Belgium
}

$\mathrm{T}$ HE ARTICle OF UTENS AND HER COLLEAGUES, published in this issue, ${ }^{1}$ draws attention to the psychosocial aspects of our profession. It is clear that the ultimate goal of medicine is not only adequate diagnosis and restoration to health, which can be equated to cure, but the total well-being and well-feeling of the individual and his or her full integration in society as an adult. Good medicine should be defined as the ideal mixture of cure, care, counselling and communication, a combination which can be called the "rule of the four C's". For too long, modern medicine has focussed exclusively on cure. Of course, the quality of cure, the first "c", is in itself a very important psychosocial factor, in the sense that the likeliness to develop problems of adjustment will decrease when an ideal cure is achieved without sequels or residual lesions. The quality of our knowledge, and our art of healing, always comes first. Without it, the three other aspects will count for little. But the other three components are equally important, not only for those seeking to practise as good doctors, but also for those who seek to guarantee the satisfaction and faith or confidence of the patients and their families in medicine and those who provide it. Care has two aspects. The first is the application of knowledge to the benefit of the patients. The second is the health services provided to them. Counselling is the professional activity of giving advice or opinion to help someone to understand his or her problems with adjustment. Communication is the exchange of information, ideas, and feelings between the takers and providers of care

We all know, nonetheless, that the final goal of good medicine is often not attained when, for

Correspondence to: Henri A. Verhaaren, Dept Paediatric Cardiology, University Hospital, De Pintelaan 185, B-9000 Ghent, Belgium. Tel: 00329240 2443; Fax: 00329240 3856; E-mail: henri.verhaaren@ rug.ac.be

Accepted for publication 13 December 2000 educational, social, or psychological reasons, the patient does not find peace with his or her scar, with his or her status as an operated person, or with the reactions of the environment to her or his abnormality. For more than twenty years, the working group of the Association for European Paediatric Cardiology concerned with psychosocial problems has tried to bring together parents, patients, teachers, psychologists, social workers, and medical professionals. Five major fields have been explored. First, the organisational aspects of medical cure and care. The second field is quality of life. The third object of study is the process of coping with disease. Since 1995 , our attention has turned to two new aspects: the ethical and the legal problems.

The publication of the investigation of Utens and her colleagues ${ }^{1}$ reflects our growing concern about the incompleteness of our medical performances. But we are faced with questions that cannot easily be assessed with our arsenal from clinical science. Psychosocial aspects of disease have, for too long, been considered the outcasts of medicine. This has resulted in a lack of published material in this field. It has been discussed in the back yards, not in the amphitheatre.

Physicians feel that they have neither the language nor competence to assess psychosocial problems without help from social and psychological sciences. Some caution, and some scepticism, with the results of such studies are warranted. Neither the social nor psychological sciences are using our usual scientific framework. Social sciences, ethical studies, law, history, even philosophy and theology, may all be important in the full understanding of the psychosocial components of disease. Clinical psychology is only one of our partners needed to help us to attain, within a multidisciplinary framework, the goal of a more complete medical approach. Furthermore, many 
other schools exist in psychology which can provide valuable insights in the world of our patients and their environment. For all these reasons I would state, somewhat provocatively, that clinical psychology is by no means the final answer to our questions.

I have the impression that the submission from Utens and her colleagues ${ }^{1}$ was accepted partly because it is born from a rather statistical approach. And the statistical methods seem rather familiar to the clinical scientist. The use of statistical methods and testing in psychology, however, is much more complex than in clinical medicine. Tests should be declared valid only in the populations in which they have been validated. Cultural, linguistic, and other reasons oblige us to make population-validated tests. When considering the statistical analysis of the results of testing, the problems become even more complicated. Often the clinical psychologist works with models. But models are worthless if not all factors are included, which often is not the case. Moreover, the population changes continuously. The very process of conducting a study in itself already changes the situation, perception, feelings, motivations, and so on. The validity of a model is only guaranteed when all variables are included, and the model stays stable over the period of study. These prerequisites are seldom guaranteed.

The main conclusion of the investigation carried out by Utens and her colleagues ${ }^{1}$ is that, in children with normal intelligence under the age of 7 , no major behavioural or emotional dysfunction was observed in the period of preparation prior to interventions. This means that, in this age group, the maternal anxiety, which is certainly present, does not result in dysfunction of the child. This is, as correctly pointed out in the discussion, opposite to the findings of the same authors in older children. This brings us to a very important point. Every study in clinical psychology, and not only in this science, is limited by the characteristics of the population examined, and its conclusions are only valid within that same population. Every paediatric cardiologist knows from daily practice that many problems of adjustment are typical for puberty and young adolescence. They disappear once the patient has become an adult. And, as an adult, such problems in adjustment can reappear when a sudden deterioration is experienced in the physical condition.

We must be very cautious when using statistics in the field of psychosocial medicine. It had long been believed that gastric ulcer was primarily a psychosomatic disease, until this was disproved by the discovery of the Helicobacter bacillus.
Correlation or association is no proof of a cause and effect relationship. Use of statistics in the field of psychosocial aspects of medicine is also limited because feelings and motivations, such as happiness, coping, enthusiasm, and many others, are not really quantifiable.

As in clinical medicine, we should stick to the very straight forward principles of good statistics defined by Bradford-Hill, the father of medical statistics, clearly summarised recently by James Le Fanu in his timeless book 'The rise and fall of modern medicine'2 These principles are:

- the correlation must be plausible

- the correlation must be strong enough (is $\mathrm{p}>.05$ sufficient?)

- the correlation must reflect a gradient so that, if the factor is stronger, the effect increases

- the correlation must be found consistently

- the correlation must hold over time

- the association has to be confirmed by experiment.

This last principle is very important. Statistical significance in itself does not prove anything in psychosocial medicine. Observing the changes after a shift in policy, which is itself a psychological or social experiment, is a much better proof than just bare statistics. As an example, we can cite the introduction of rooming-in. Before this change in the policy of hospitals, it would be hard to prove statistically that this simple change would result in the disappearance of the disease, with nightmares, depression, and so on, from which children were suffering in the weeks after their stay in hospital.

The publication of a paper on psychosocial problems in paediatric cardiology is a good occasion with which to focus on our common lack of knowledge in this particular field. Here, we all need continuous education and ongoing research. Psychosocial research is by definition a multidisciplinary endeavour, and we all need education in the possibilities and limitations of the social and psychological sciences we consult in these matters. I can only hope that Cardiology in the Young will continue to take the challenge to bridge this gap. I would further suggest that the editors should not accept only 'statistical' papers concerned with clinical psychological and psychosocial aspects as the gold standard of psychosocial research. Multidisciplinary research is a much broader field of action. To conclude, we should not forget the end-point of all these endeavours: a better and happier life for our patients. 
Vol. 11 , No. 2

\section{References}

1. Utens EMWJ, Versluis-Den Bieman HJ, Witsenburg M, Bogers AJJC, Verhulst FC, Hess J. Cognitive and behavioral/emotional functioning of young children awaiting elective cardiac surgery or cardiac catheter intervention. Cardiol Young 2001;2: 153-160

2. James Le Fanu. The Rise and Fall of Modern Medicine.

Paperback edition, Abacus, London, 2000: pp. 58-59 